\title{
INTRODUCCIÓN AL DISEÑO DE PERFIL DE LEVAS POR CURVAS DE BEZIER
}

\author{
Carlos Acevedo Peñaloza \\ Doctorando. M.Sc. Prof. Universidad Francisco de Paula \\ Santander. Colombia. \\ carlos.humberto.acevedo@upc.es \\ Enrique Zayas Figueras \\ Doctor. Prof Universidad Oscar Lucero Moya. Cuba \\ Salvador Cardona i Foix \\ Doctor. Prof. Universidad Politécnica de Cataluña. España. \\ salvador.cardona@upc.es
}

\section{RESUMEN}

En el estudio de la ley de desplazamiento de los mecanismos leva-palpador se puede utilizar cualquier tipo de función, comúnmente se ha utilizado funciones polinómicas, sinusoidales y cíclicas. Los métodos tradicionales utilizan polinomios algebraicos con base canónica y polinomios trigonométricos con base Fourier, siendo estos los encontrados en casi todos los textos.

La novedad del presente trabajo, esta en la utilización para el diseño de la ley de desplazamientos de Curvas Bezier no paramétricas definidas por polinomios algebraicos con base de Bernstein.

Palabras claves:

Bezier, Levas, Bernstein.

\section{ABSTRACT}

For the study of the law of displacement, any type of function can be used. Commonly it has been used polynomial, sinusoidal and cyclic functions. The traditional methods utilize algebraic polynomials with canonical base and trigonometric polynomials with Fourier base, being these used in almost all the texts.

The importance of the present work is the utilization of the non-parametric Bezier's curves defined by algebraic polynomials with Bernstein base for the law of displacement.

Key words:

Bezier, Cams, Bernstein.

\section{INTRODUCCIÓN}

Algunos autores entre los que se puede mencionar a Erdman y Sandor (1998), Norton, R. (2002), Shigley y Uicker (1998), Mabie y Reinholtz (2000) y Cardona y Clos (2000), exponen el proceso de diseño de los mecanismos leva palpador, pero son los dos últimos quienes describen claramente tres etapas para tal labor: -definición de la ley de movimiento del palpador, -obtención del perfil de la leva y por último comprobación del perfil estos autores muestran además, las técnicas de diseño de curvas por Bezier

La mayor parte de la bibliografía básica consultada trata la definición de la ley de desplazamiento del palpador, con los denominados métodos tradicionales, los cuales utilizan polinomios algebraicos con base canónica y polinomios trigonométricos con base de Fourier.

Los polinomios algebraicos expresados con base canónica o monomial, han tenido amplio uso en la definición de la ley de desplazamiento debido a que son fáciles de manipular. Esta base para grado $n$ consiste en los monomios: $\left[1, \theta, \theta^{2}, \ldots . ., \theta^{n}\right]$ La expresión de la ley de desplazamiento s $(\theta)$ en esta base es: (Reyes, Zayas)

$$
s(\theta)=\sum_{i=1}^{n} a_{i} \theta^{i}=a_{0}+a_{1} \theta+a_{2} \theta^{2}+\ldots+a_{n} \theta^{n}
$$

La expresión de un polinomio trigonométrico en la base Fourier es:

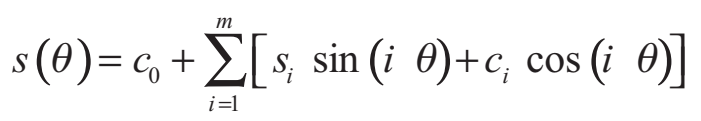

Donde $a_{i}, c_{i}$, y $s_{i}$, son los coeficientes de los polinomios anteriores.

Curva de Bezier no paramétrica.

La definición de la ley de desplazamiento del palpador por medio de la base de polinomios de Bernstein presenta ventajas en lugar de la base de monomios. Los polinomios de Bernstein de grado $n, B_{i}^{n}(u)$ sobre un dominio unitario son: 
Introducción al diseño de perfil de Levas por curvas de Bezier

Carlos Acevedo Peñaloza, Enrique Zayas Figueras y Salvador Cardona i Foix

$$
\begin{array}{r}
B_{i}^{n}(u)=\left(\begin{array}{c}
n \\
i
\end{array}\right) u^{i}(1-u)^{n-i}=C_{n}^{i} u^{i}(1-u)^{n-i} \\
i=0, \ldots ., n
\end{array}
$$

Donde

$$
\begin{gathered}
\left(\begin{array}{c}
n \\
i
\end{array}\right)=C_{n}^{i}=\frac{n !}{i !(n-i) !} ; \\
B_{i}^{n}(u)=0 \\
i \notin\{0, \ldots ., n\}
\end{gathered}
$$

Estos polinomios presentan las siguientes propiedades:

-Satisfacen la formula recursiva.

$i \in\{0, \ldots ., n\}: B_{i}^{n}(u)=(1-u) B_{i}^{n-1}(u)+u B_{i-1}^{n-1}(u)$,

con $B_{0}^{0}=1$

-Son una partición de la unidad

$\sum_{i=0}^{n} B_{i}^{n}(u)=1$

-Positividad: $\quad B_{i}^{n}(u) \geq 0, \quad u \in[0,1]$

-Simetría: $B_{i}^{n}(u)=B_{n-i}^{n}(1-u)$

-Presentan un máximo para la abscisa:

$$
u=\frac{i}{n}
$$

- Constituye una base numérica estable.

En esta base una función polinómica $b(u)$ de grado $n$ se expresa de la siguiente manera:

$$
b(u)=\sum_{i=0}^{n} b_{i} B_{i}^{n}(u) \quad u \in[0,1]
$$

Donde los $n+1$ coeficientes se denominan ordenadas de Bezier. El gráfico de la función $b(u)$ se llama curva de Bezier no paramétrica. Cada ordenada $b_{i}$ define un punto $b_{i}$ de coordenadas $b_{i}=\left(1 / n, b_{i}\right)$ denominado punto de control y dada su definición se encuentran equiespaciados en el eje de las abscisas.

En la ecuación (1.4) el polinomio $B_{i}^{n}(u)$ se puede interpretar como la influencia $b_{i}$ de en la curva $b(u)$. Esta influencia es máxima para $u=1 / n$ donde $B_{i}^{n}(u)$ presenta su máximo valor, coincidiendo con la abscisa del punto $b_{i}$ esto hace que la representación gráfica de la curva tienda a ser próxima a la del polígono de control de la figura. Por lo tanto, al desplazar verticalmente un punto $b_{i}$ modificando el valor de su ordenada, la curva tiende a seguirlo deformándose en sus proximidades.

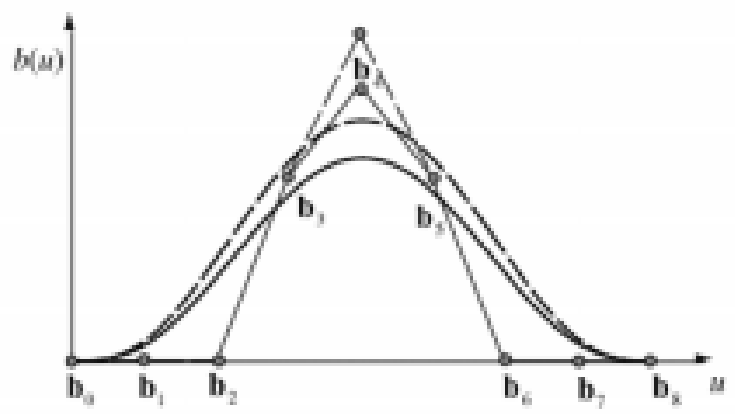

Fig. 1.1 Curva de Bezier. Tomado de Zayas 2001.

Se observa que:

- La curva pasa por los puntos extremos $b_{0}, b_{n}$ y es tangente al polígono de control en estos puntos.

- La curva se encuentra encerrada dentro del dominio convexo de los puntos de control. El polígono de control permite establecer una caja contenedor dentro de la cual se puede asegurar que se encuentra la curva.

Estas cualidades le permiten al diseñador adaptar las especificaciones de la ley de desplazamiento a sus necesidades. 
Introducción al diseño de perfil de Levas por curvas de Bezier

Carlos Acevedo Peñaloza, Enrique Zayas Figueras y Salvador Cardona i Foix

Para definir una función $b(\theta)$ con la variable independiente $\theta$ definida en el dominio no unitario $\theta \in\left[\theta_{l}, \theta_{f}\right]$ con la utilización de las curvas de Bezier $b(u)$ definidas en un dominio unitario $u \in[0,1]$, es necesario realizar en la ecuación (1.4) el cambio lineal de variable siguiente:

$$
\begin{gathered}
\theta \in\left[\theta_{i}, \theta_{f}\right] \rightarrow u \in[0,1] \\
u(\theta)=\frac{\theta-\theta_{i}}{\theta_{f}-\theta_{i}}
\end{gathered}
$$

Usualmente es necesario la derivada de $b(\theta)$ respecto a $\theta$, en cuyo caso esta se calcula:

$$
\frac{d b(u(\theta))}{d \theta}=\frac{d b(u)}{d u} \mathrm{~g} \frac{d u(\theta)}{d \theta}=\frac{d b(u)}{d u} \mathrm{~g} \frac{1}{\theta_{f}-\theta_{i}}
$$

La derivada $b^{\prime}(u)$ de un polinomio $b(u)$ de grado $n$ es un polinomio de grado $n^{\prime}=n-1$. En la base Bernstein se puede comprobar que los coeficientes $b_{i}^{\prime}$ de la derivada se obtienen como:

$$
\begin{gathered}
b_{i}^{\prime}=n\left(b_{i+1}-b_{i}\right) \\
i=0, \ldots, n-1
\end{gathered}
$$

Esto indica que las derivadas r-ésimas en los extremos $u=0, u=1$ solo dependen de los $r+1$ puntos de control mas próximos, es decir, en $u=O$ sólo depende de los coeficientes $b_{O} . . . b_{r}$. y en $u=1$ de los coeficientes $b_{n-r . . .} b_{n}$. Por lo tanto, la imposición de condiciones de continuidad r-ésima en la unión entre dos curvas resulta más sencilla en la base de Bernstein que en la base canónica o monomial, ya que solo se ven involucrados $r+1$ coeficientes de cada curva.

Debido a esto, las curvas de Bezier no paramétrica constituyen una buena herramienta para generar las curvas de desplazamiento, velocidad, aceleración y sobre aceleración de trabajo en levas.
En la figura 1.2 se indica a manera general el uso de los puntos de control, el manejo de la continuidad en las curvas, así como el polígono de control.

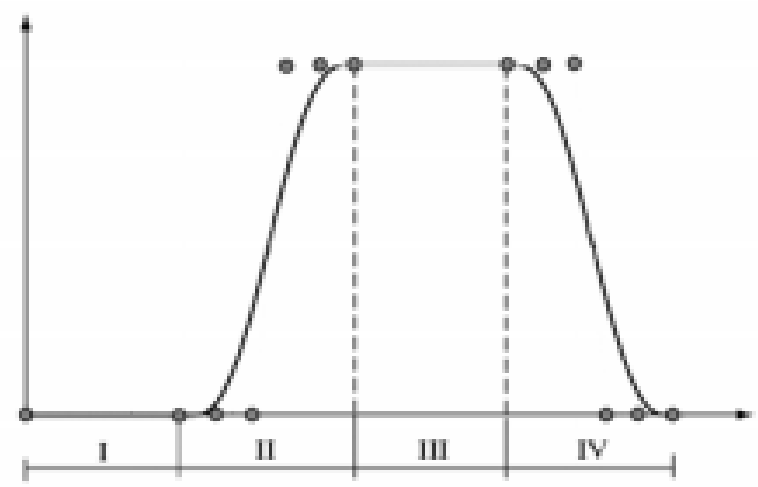

Fig. 1.2 Los puntos de control en la función desplazamiento. Tomado de Zayas 2001.

\section{MÉTODO ANALÍTICO DE OBTENCIÓN DE PERFIL DE LEVAS.}

A continuación se muestra el procedimiento analítico de obtención de perfil de levas de placa plana con seguidor de rodillo en traslación basado en el enfoque Cardona y Clos. No se muestra el procedimiento de cálculo por el método tradicional por encontrarse en casi todos los textos de teoría de máquinas y mecanismo.

Cuando los palpadores tienen movimiento de traslación, la ley general $d(\theta)$ de desplazamiento es:

$$
d(\theta)=s(\theta)+d_{0}
$$

donde $s(\theta)$ es la ley de desplazamiento especificada con métodos tradicionales y $d_{O}$ ver figura 1.3 es un parámetro que en el caso de palpadores planos depende del radio base $R_{b}$ de la leva y del valor de la excentricidad ' $\varepsilon$ del palpador. Para el palpador circular, el parámetro $d_{O}$ que interviene en la función es:

$$
d_{0}=\sqrt{\left(R_{b}+R_{r}\right)^{2}-\varepsilon^{2}}
$$


Introducción al diseño de perfil de Levas por curvas de Bezier

Carlos Acevedo Peñaloza, Enrique Zayas Figueras y Salvador Cardona i Foix

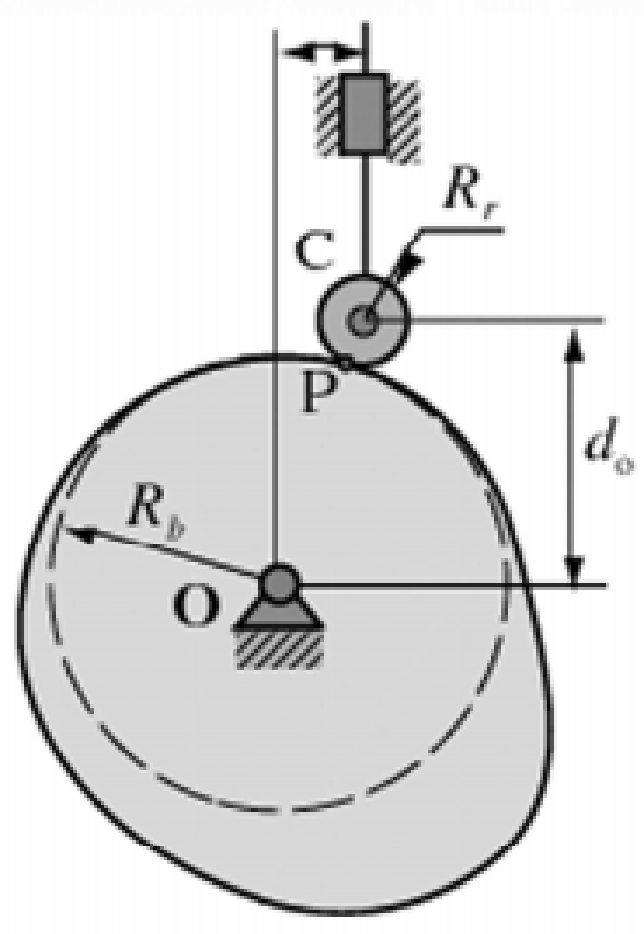

Fig. 1.3 Descripción general de la leva. Tomado de Zayas 2001.

En el caso de un palpador circular de centro $C$ y radio $R_{r}$, el perfil de la leva es la envolvente de un haz de circunferencia. La trayectoria que describe el centro de esa circunferencia es la curva de paso y su posición se calcula:

$$
\{\overline{O C}(\theta)\}_{1,2}=\left\{\begin{array}{c}
\varepsilon \\
d(\theta)
\end{array}\right\}_{1,2}
$$

En la figura 1.4 se puede ver claramente el desarrollo de la ecuación

En la base fija $x, y$ la ecuación paramétrica de la curva de paso es:

$$
\{\overline{O C}(\theta)\}_{X, Y}=\left[S_{\theta}\right]\{\overline{O C}(\theta)\}_{1,2}=\left\{\begin{array}{c}
\varepsilon \cos \theta+d(\theta) \sin \theta \\
-\varepsilon \sin \theta+d(\theta) \cos \theta
\end{array}\right\}_{x, y}
$$

El perfil de la leva $\overline{O P}(\theta)$, equivale al lugar geométrico de los puntos situados a una distancia $R_{r}$ de la curva de paso en la dirección de su normal $n(\theta)$ interior, y dirigida hacia el área que encierra la curva. La ecuación paramétrica del perfil es:

$$
\overline{O P}(\theta)=\overline{O C}(\theta)+R_{r} \cdot n(\theta)
$$

Para calcular la normal unitaria interior a la curva de paso, $n(\theta)$, se necesita el vector tangente a la curva $t(\theta)$ que se calcula:

$t(\theta)=\frac{d}{d \theta} \overline{O C}(\theta)=\left\{\begin{array}{l}\left(d^{\prime}(\theta)-\varepsilon\right) \sin \theta+d(\theta) \cos \theta \\ \left(d^{\prime}(\theta)-\varepsilon\right) \cos \theta-d(\theta) \sin \theta\end{array}\right\}$

Entonces:

$$
n(\theta)=\left[R_{-90^{\circ}}\right] \frac{t(\theta)}{|t(\theta)|}
$$

Donde $\left[R_{-90^{\circ}}\right]$ es la matriz de rotación:

$$
\left[\begin{array}{cc}
0 & 1 \\
-1 & 0
\end{array}\right]
$$

Para la obtención radio de curvatura, se deriva la expresión 1.10 respecto al parámetro $\theta$ en la base móvil 1,2:

$$
\begin{aligned}
& \{\overline{O C}(\theta)\}_{1,2}=\left\{\begin{array}{c}
e \\
d(\theta)
\end{array}\right\}_{X, Y} \stackrel{d / d \theta}{\longrightarrow}\left\{\begin{array}{c}
d(\theta) \\
d^{\prime}(\theta)-\varepsilon
\end{array}\right\}_{X, Y} \\
& \stackrel{d / d \theta}{\longrightarrow}\left\{\begin{array}{c}
2 d^{\prime}(\theta)-\varepsilon \\
d^{\prime \prime}(\theta)-d(\theta)
\end{array}\right\}_{X, Y}
\end{aligned}
$$


Introducción al diseño de perfil de Levas por curvas de Bezier

Carlos Acevedo Peñaloza, Enrique Zayas Figueras y Salvador Cardona i Foix
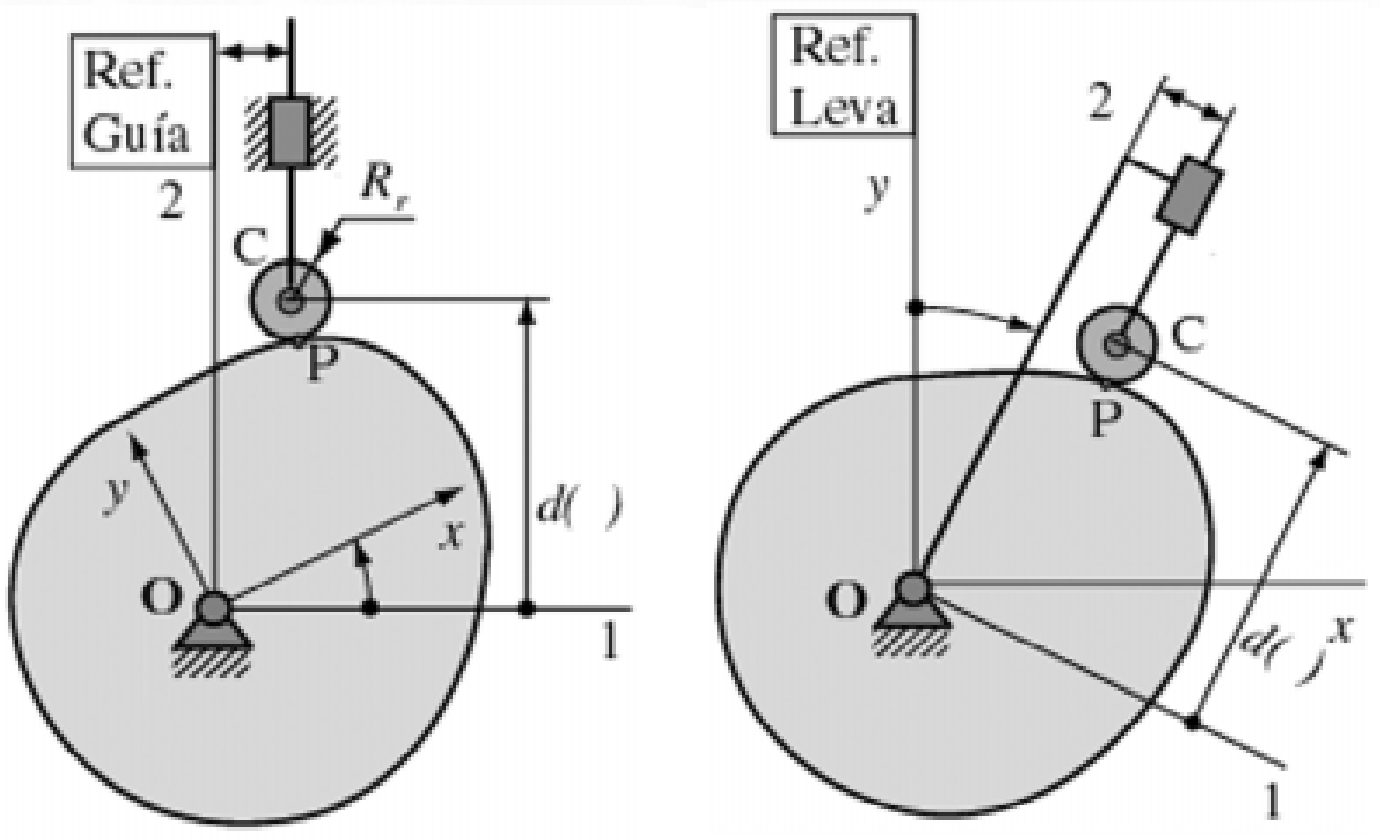

Fig. 1.4 Rotacional de la Leva

Como se ha parametrizado en sentido horario de manera que la componente normal (hacia el interior) de la derivada se expresa como:

$\left.\overrightarrow{O C^{\prime \prime}}\right|_{n}(\theta)=\frac{\overline{O C^{\prime \prime}}(\theta) \times\left.\overline{O C^{\prime}}\right|_{e j 33}}{\left|\overrightarrow{O C^{\prime}(\theta)}\right|}$

sustituyendo la ecuación 1.15 y 1.16 en

$$
\begin{gathered}
r_{c}=\frac{\left|\overline{O C^{\prime}}\right|^{2}}{\left.O P^{\prime \prime}\right|_{n}} \text { se tiene: } \\
r_{c p}(\theta)=\frac{\left(d^{2}(\theta)+\left(d^{\prime}(\theta)-\varepsilon\right)^{2}\right)^{3 / 2}}{\left(d^{\prime}(\theta)-\varepsilon\right)\left(2 d^{\prime}(\theta)-\varepsilon\right)-d(\theta)\left(d^{\prime \prime}(\theta)-d(\theta)\right)}
\end{gathered}
$$

que es equivalente a la ecuación que aparece en los libros que usan métodos tradicionales:

$$
\rho=\frac{\left[\left(R_{0}+s\right)^{2}+\left(\frac{d s}{d \theta}\right)^{2}\right]^{3 / 2}}{\left(R_{0}+s\right)^{2}+2\left(\frac{d s}{d \theta}\right)^{2}-\left(R_{0}+s\right)\left(\frac{d^{2} s}{d \theta^{2}}\right)}
$$

\section{CONCLUSIONES}

Es poco favorable utilizar polinomios con base canónica o con base Fourier, para diseñar las funciones de desplazamiento de las levas puesto que presentan las siguientes desventajas:

- Los coeficientes $a_{i}$ de la ecuación (1.1) y $c_{i}$ y $s_{i}$ de la ecuación (1.2), no tienen significado geométrico, por lo tanto la modificación de un determinado coeficiente no produce un efecto intuitivo sobre la forma de la función $s(\theta)$, resultando dificil gobernar la curva para obtener unas características determinadas.

- Los coeficientes de los polinomios se obtienen al solucionar un sistema de ecuaciones que se origina 
Introducción al diseño de perfil de Levas por curvas de Bezier

Carlos Acevedo Peñaloza, Enrique Zayas Figueras y Salvador Cardona i Foix

de las restricciones que debe cumplir $s(\theta)$. Debido a estos coeficientes es engorroso automatizar la aproximación porque cada caso requiere un tratamiento particular.

- La base canónica de polinomios algebraicos no garantiza estabilidad numérica, especialmente si el grado $n$ del polinomio es elevado.

- La imposición de las condiciones de continuidad en la unión entre los tramos descenso, subida y bajada de $s(\theta)$ resulta laborioso.

\section{BIBLIOGRAFÍA}

Erdman, A. Sandor, G. Diseño de Mecanismos, Análisis y Síntesis. 1998

Cardona, S. Clos, D. Teoría de Máquinas. 2000.

Mabie y Reinholtz. Mecanismos y Dinámica de maquinaria. 2000.

Norton, R. Cam Design and manufacturing, handbook. 2002.

Reyes, G. Técnicas de Diseño Geométrico Asistido por Ordenador para Mecanismo Leva-Palpador. 2000.

Shigley y Uicker. Teoría de Máquinas y Mecanismos. 1988.

Zayas, E. Aportación al Estudio de Levas Desmodrómicas. 2001. 